\title{
X-ray properties of active $M$ dwarfs as observed by XMM-Newton
}

\author{
J. Robrade and J. H. M. M. Schmitt
}

\author{
Hamburger Sternwarte, Universität Hamburg, Gojenbergsweg 112, 21029 Hamburg, Germany \\ e-mail: jrobrade@hs.uni-hamburg.de
}

Received 2 September 2004 / Accepted 8 February 2005

\begin{abstract}
We present a comparative study of X-ray emission from a sample of active M dwarfs with spectral types M3.5-M4.5 using XMM-Newton observations of two single stars, AD Leonis and EV Lacertae, and two unresolved binary systems, AT Microscopii and EQ Pegasi. The light curves reveal frequent flaring during all four observations. We perform a uniform spectral analysis and determine plasma temperatures, abundances and emission measures in different states of activity. Applying multi-temperature models with variable abundances separately to data obtained with the EPIC and RGS detectors we are able to investigate the consistency of the results obtained by the different instruments onboard XMM-Newton. We find that the $\mathrm{X}$-ray properties of the sample $\mathrm{M}$ dwarfs are very similar, with the coronal abundances of all sample stars following a trend of increasing abundance with increasing first ionization potential, the inverse FIP effect. The overall metallicities are below solar photospheric ones but appear consistent with the measured photospheric abundances of $\mathrm{M}$ dwarfs like these. A significant increase in the prominence of the hotter plasma components is observed during flares while the cool plasma component is only marginally affected by flaring, pointing to different coronal structures. AT Mic, probably a young pre-main-sequence system, has the highest X-ray luminosity and exhibits also the hottest corona. While results of EQ Peg and EV Lac are presented here for the first time, AT Mic and AD Leo have been investigated before with different analysis approaches, allowing a comparison of the results.
\end{abstract}

Key words. stars: activity - stars: coronae - stars: flare - stars: late-type - X-rays: stars

\section{Introduction}

Observations with ROSAT have shown the formation of X-ray emitting coronae around late-type cool dwarf stars with outer convection zones to be universal (Schmitt et al. 1995; Schmitt $\&$ Liefke 2004). Coronal structures on the Sun are dominated by their magnetic properties with closed structures, the coronal loops or loop arcades, containing predominantly the $\mathrm{X}$-ray emitting, confined hot high-density plasma and being the location of flare events. In the Sun the interaction between the radiative and the outer convective zones powers a dynamo, leading to coronal activity. The ubiquitous occurrence of X-ray emission among cool stars and their dependence on rotation strongly suggests also a magnetic character of their activity. Observed activity levels of cool stars, as measured by the ratio of bolometric and $\mathrm{X}$-ray luminosities, span a range of values from $\sim 10^{-8}$ to $\sim 10^{-3}$ that are correlated with dynamo efficiency. The efficiency of a solar-like magnetic dynamo is characterised by the inverse of the so-called "RossbyNumber", defined as the ratio between rotational period and convective turnover time. This activity-rotation relation saturates at $L_{\mathrm{X}} / L_{\mathrm{bol}} \sim 10^{-3}$, a level $>1000$ times higher than that of the Sun. The similarity of stellar and solar X-ray emission and flares suggests similar basic physical mechanisms, with more active stars showing more frequent and larger flares. Flaring has been proposed as a possible major coronal heating mechanism for the Sun via nanoflares (Parker 1988) as well as for other cool stars including $\mathrm{M}$ dwarfs. For recent reviews on this topic, see Kashyap et al. (2002) or Güdel et al. (2003).

Active $\mathrm{M}$ dwarfs, belonging to the so-called flare stars, turned out to be particularly strong coronal X-ray sources. Frequent flaring on these objects was first discovered in the optical regime, later in EUV and X-rays. These late-type dwarf stars are generally more active than solar-like stars since they have larger spin-down times. They also typically show higher coronal temperatures. $\mathrm{M}$ dwarfs of spectral types later than approximately M3-M5 are expected to be fully convective and therefore lack an interface between radiative and convective zones. The observed high levels of activity then require the onset of alternative dynamo mechanisms different to that of the Sun.

The stars chosen here for analysis are known high X-ray emitters with spectral types in the range M3.5-M4.5 and are located in the immediate solar vicinity (distance 5-10 pc). EQ Peg (Gl 896A, Gl 896B) is a visual binary consisting of two M dwarfs of spectral type M3.5 and M4.5, both of which are flare stars, as well as AT Mic (Gl 799A, Gl 799B), an active binary system consisting of a M4.5 and a M4 star. Both binaries are separated by roughly $30 \mathrm{AU}$, making interaction between the components quite unlikely. On the other hand, AD Leo (Gl 388) is a single M3.5 star similar to EV Lac (Gl 873). Our sample M dwarfs are also all fast rotators 
Table 1. Stellar parameters; $v \sin i$ from Delfosse et al. (1998) except AT Mic (Fuhrmeister et al. 2004) and EQ Peg (Stauffer \& Hartmann 1986), $J$ from 2 MASS point source catalog, $L_{\mathrm{bol}}$ calculated from $J$ using values of Kenyon \& Hartmann (1995), $L_{\mathrm{bol}} / L_{\mathrm{X}}$ with $L_{\mathrm{X}}$ (this work, PN).

\begin{tabular}{lccccc}
\hline \hline Star & $d(\mathrm{pc})$ & $v \sin i\left(\mathrm{~km} \mathrm{~s}^{-1}\right)$ & $J(\mathrm{mag})$ & $\log L_{\mathrm{bol}}$ & $\log L_{\mathrm{bol}} / L_{\mathrm{X}}$ \\
\hline AT Mic A+B & 10.2 & $11.7(\mathrm{~A})$ & 5.81 & 32.46 & -3.00 \\
EQ Peg A+B & 6.2 & $12 / 18(\mathrm{~A} / \mathrm{B})$ & 5.79 & 32.04 & -3.22 \\
AD Leo & 4.7 & 6.2 & 5.45 & 31.94 & -3.21 \\
EV Lac & 5.0 & 6.9 & 6.11 & 31.73 & -2.99 \\
\hline
\end{tabular}

$\left(v \sin i>5 \mathrm{~km} \mathrm{~s}^{-1}\right.$ ) (Delfosse et al. 1998; Fuhrmeister et al. $2004)$ in the saturated regime, i.e. $L_{X} / L_{\text {bol }} \sim 10^{-3}$. Stellar parameters important for this work are summarized in Table 1. Note that the spectral classification in the literature is not unique for all $\mathrm{M}$ dwarfs in our sample and may differ by one spectral subclass; the values listed here are taken from the SIMBAD database.

While X-ray emission from AD Leo and AT Mic was already detected by HEAO 1 (Kahn et al. 1979) and EQ Peg is contained in the Einstein Stellar Survey (Vaiana et al. 1981), EV Lac was first detected by EXOSAT (Schmitt \& Rosso 1988). All stars in our sample were also detected in the ROSAT all-sky survey (Hünsch et al. 1999) and were observed by various other X-ray missions. A comparative analysis of X-ray spectra from AD Leo and EV Lac obtained with BeppoSAX and ROSAT PSPC was performed by Sciortino et al. (1999). Favata et al. (2000a) presented a study of an extreme X-ray flare on EV Lac observed with ASCA. Favata et al. (2000b) studied AD Leo data taken by Einstein, ROSAT and ASCA and found the coronal structures to be compact and the quiescent coronal luminosity remarkably constant over decades.

Parts of the XMM-Newton data of AT Mic were previously analysed with special focus on elemental abundances and emission measures during flaring and quiescent state (Raassen et al. 2003). While the coronae of low activity stars like the Sun (Laming et al. 1995) show an enhancement of low-FIP elements, the so-called FIP (First Ionization Potential) effect, Raassen et al. (2003) discovered in the corona of the highly active star AT Mic an inverse FIP effect, which appears to flatten during the flare state. A comparative study of flares on AD Leo observed with XMM-Newton and Chandra was performed by Besselaar et al. (2003) and again an inverse FIP effect was found. An analysis of spatially resolved X-ray emission of the EQ Peg system was presented by Robrade et al. (2004). A comparative study on coronal density diagnostics with He-like triplets that includes work on our sample stars can be found in Ness et al. (2002) and with special focus on the X-ray sizes of coronae in Ness et al. (2004).

The above listed $\mathrm{M}$ dwarfs were observed with XMM-Newton and the goal of this paper is to present a comparative analysis of these X-ray observations and to determine those properties that are typical for active $M$ dwarfs as a class and those attributed to individual objects. In Sect. 2 we describe the observations and the methods used for data
Table 2. Observation log.

\begin{tabular}{llcc}
\hline \hline Target & Instrument (mode) & Dur. (s) & Obs. time \\
\hline EQ Peg & MOS (FF, thick F.) & 14600 & 2000-07-09T11:39-15:43 \\
& PN (FF, thick F.) & 12410 & 2000-07-09T12:20-15:47 \\
\hline & RGS (HER/SES) & 15610 & 2000-07-09T11:31-15:51 \\
\hline AT Mic & MOS1 (SW, med. F.) & 25400 & 2000-10-16T00:28-07:32 \\
$"$ & PN (SW, med. F.) & 25100 & 2000-10-16T00:42-07:40 \\
$"$ & RGS (HER/SES) & 28310 & 2000-10-16T00:20-08:11 \\
\hline AD Leo & MOS2 (LW, thick F.) & 35800 & 2001-05-14T20:55-06:52 \\
& PN (SW, med. F.) & 35000 & $2001-05-14 T 21: 11-06: 54$ \\
$"$ & RGS (HER/SES) & 36400 & $2001-05-14 T 20: 48-06: 55$ \\
\hline EV Lac & MOS2 (SW, med. F.) & 32260 & $2001-06-03 T 07: 55-16: 53$ \\
$"$ & PN (SW, med. F.) & 31470 & $2001-06-03 T 08: 11-16: 55$ \\
$"$ & RGS (HER/SES) & 32860 & $2001-06-03 T 07: 49-16: 56$ \\
\hline
\end{tabular}

analysis. In Sect. 3 we present the results subdivided into different physical topics which is followed by a summary and our conclusions in Sect. 4.

\section{Observation and data analysis}

The four targets, AD Leo, AT Mic, EV Lac and EQ Peg, were observed with XMM-Newton using varying detector setups and exposure times in the range of $15-35 \mathrm{ks}$. No attempts were made to separate the binaries in this work, therefore data from AT Mic and EQ Peg is a superposition of the respective system components. A detailed description of the observations is provided in Table 2. Useful data were collected in all X-ray detectors onboard XMM-Newton, respectively the EPIC (European Photon Imaging Camera) and RGS (Reflection Grating Spectrometer) detectors which are operated simultaneously. The EPIC instrument consists of three CCD cameras with two different CCD designs, two MOS and one PN, providing imaging and spectroscopy in the energy range from 0.15 to $15 \mathrm{keV}$ with good angular and moderate spectral resolution. In this work EPIC data is used only when the detectors observed in the imaging mode and therefore data from both MOS detectors are presented only for EQ Peg. The RGS is a grating spectrometer with high spectral resolution consisting of two identical spectrometers. The RGS operates in the energy range from 0.35 to $2.5 \mathrm{keV}(5-35 \AA)$ with a spectral resolution of $\sim 4.0 \mathrm{eV}(0.06 \AA, F W H M)$ at $1.0 \mathrm{keV}$ in first order. A detailed description of the XMM-Newton instruments is given by Ehle et al. (2003).

The data were reduced with the standard XMM-Newton Science Analysis System (SAS) software, version 5.4.1 with calibration files updated in January 2004. Light curves and spectra were produced with standard SAS tools and standard selection criteria were applied for filtering the data (see Ehle et al. 2004). Spectral analysis was done with XSPEC V11.3 (Arnaud 1996).

Data from the detectors of different type were fitted separately to allow comparison of the results and to check for possible cross-calibration problems. Spectral analysis 
of EPIC data is performed in the energy band between $0.2-12.0 \mathrm{keV}$. RGS first order spectra in the full energy range, i.e. $0.35-2.5 \mathrm{keV}(5-35 \AA)$ are used. Data of the same detector type, e.g. RGS1 and RGS2, and in the EQ Peg case MOS1 and MOS2, were analyzed simultaneously but not co-added. For spectral analysis the data was cleaned of proton flares by removing the affected time periods, and in the EQ Peg case the data was additionally cleaned for pile-up by excluding the inner part of the source emission. The applied selection criteria were adjusted according to observation mode. The background was taken from source-free regions on the detectors.

We apply the same type of spectral model to all data to perform a self-consistent analysis. Therefore we use the global fitting approach, where the whole spectrum is fitted instead of a line-based method which would be applicable only to RGS data. Audard et al. (2004) applied both methods to data of YY Mensae taken by Chandra and XMM-Newton and found consistent results. These authors fitted data from RGS and MOS in selected energy ranges simultaneously, similar to our joint fits, which are briefly discussed in Sect. 3.2.2. Larger deviations were found when using data of different detectors, a problem also affecting our analysis. However, Sanz-Forcada et al. (2003) analysed Chandra and XMM-Newton data of AB Dor, a highly active near-main-sequence $\mathrm{K}$ star, and found the line-based method to be better suited to fit their high resolution spectra, leading to slightly higher abundances and lower emission measures to be present in the derived models compared to the global fitting approach. Güdel et al. (2001) analysed EPIC and RGS spectra from different observations of AB Dor using the global approach and found different absolute abundance values but a pattern (increasing depletion of abundance of low FIP elements up to iron followed by the inverse pattern for elements with higher FIP) overall consistent with the results derived by Sanz-Forcada et al. (2003), demonstrating the robustness of abundance ratios in general.

For the analysis of the X-ray spectra we specifically use multi-temperature models with variable but tied abundances, i.e. the same abundance pattern in all temperature components. Such models assume the emission spectrum of a collisionally-ionized diffuse optically-thin gas as calculated with the APEC code (see Smith et al. 2001). Abundances are calculated relative to solar photospheric values as given by Anders \& Grevesse (1989). For iron and oxygen we use the updated values of Grevesse \& Sauval (1998). Comparing stellar coronal with solar photospheric abundances is of course crude, but precise photospheric abundances are unknown for the observed targets; a detailed discussion of the abundances is given in Sect. 3.2.3. Due to the proximity of the stars, absorption in the interstellar medium is negligible at the wavelengths of interest and was not applied in our modelling. Our fit procedure is based on $\chi^{2}$ minimization, therefore spectra are rebinned to satisfy the statistical demand of a minimum value of counts per spectral bin; 25 for data with an average count rate above $5 \mathrm{cts} \mathrm{s}^{-1}$ and 15 for data with an average count rate below $5 \mathrm{cts} \mathrm{s}^{-1}$ and for all data used in time resolved analysis. Bad channels are always excluded from the fit; these include channels at high energy in the EPIC spectra that contain insufficient source counts (see Fig. 3). The first model uses three temperature components (3-T model) with free temperatures and emission measures. Models with additional temperature components were checked, but did not improve the fit results significantly. In order to approximate physically more realistic continuous temperature distributions of the plasma, we use a 6 -T model on a logarithmic, almost equidistant grid $(0.2,0.3,0.6,1.2,2.4,4.8 \mathrm{keV})$, which covers roughly the temperature range from 2 to $60 \mathrm{MK}$ and samples those spectral regions where the XMM-Newton detectors are most sensitive.

Consider next the different spectral resolution, $\Delta E / E$, and energy range of the EPIC and RGS instruments. Especially at low energies, $\Delta E / E$ is quite large for the EPIC detectors, while in the RGS most of the strongest emission lines are separated in this energy range, thus permitting a more accurate determination of plasma parameters. Some elements, in particular C and N, show prominent features only in the RGS. Therefore, to fit the EPIC data the abundances of these elements were set to the RGS value in order to avoid unphysical solutions. For elements such as $\mathrm{Al}, \mathrm{Ca}$ and $\mathrm{Ni}$, whose abundances in the solar photosphere are an order of magnitude lower than that of Fe and which show only very weak features in the X-ray spectrum, we adopted a coronal abundance of 0.4 times the solar photospheric abundance (a typical value for $\mathrm{Fe}$ in this sample). The EPIC detectors are able to measure higher energy X-rays, important for a reliable determination of the properties of the hotter plasma components, with the MOS detectors providing a slightly better spectral resolution and the PN detector providing greater sensitivity.

An important feature of the considered spectral models is the interdependency between the emission measure $(E M=$ $\left.\int n_{\mathrm{e}} n_{\mathrm{H}} \mathrm{d} V\right)$ of temperature components on the one hand and elemental abundances with temperature sensitive emission lines in the considered temperature range on the other hand. This is especially true for EPIC data with their moderate spectral resolution. Another general source of uncertainties is the incomplete knowledge of atomic data used to calculate the spectral models, see e.g. Porquet et al. (2001) and references therein.

\section{Results}

\subsection{Investigation of light curves}

The PN light curves of the four observations are shown in Fig. 1; these light curves were extracted from a circle with 50 " radius around each source and the temporal binning is $100 \mathrm{~s}$ for all sources. Light curves were cleaned (AD Leo, AT Mic, EV Lac) for obvious data dropouts and high proton flare background was subtracted from the light curve (EV Lac). As the PN exposure of AD Leo began during a flare, we included scaled data from the MOS2 exposure, which started $\sim 1$ ks earlier, to show the peak of the flare.

The light curves of the individual targets show obvious variability in all sources. The largest flares that occurred in these observations involve X-ray flux increases of factors 2-3, which is considered moderate in view of flux increases of factors 10-300 that have been observed in flares on EQ Peg (Katsova et al. 2002) and EV Lac (Favata et al. 2000a). For an analysis of a larger flare on the $\mathrm{M}$ dwarf Proxima Centauri with 
EQ Peg

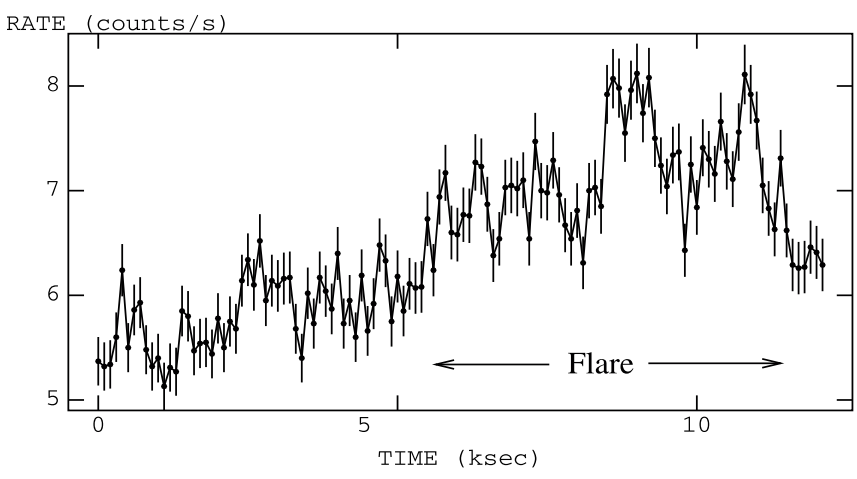

AT Mic

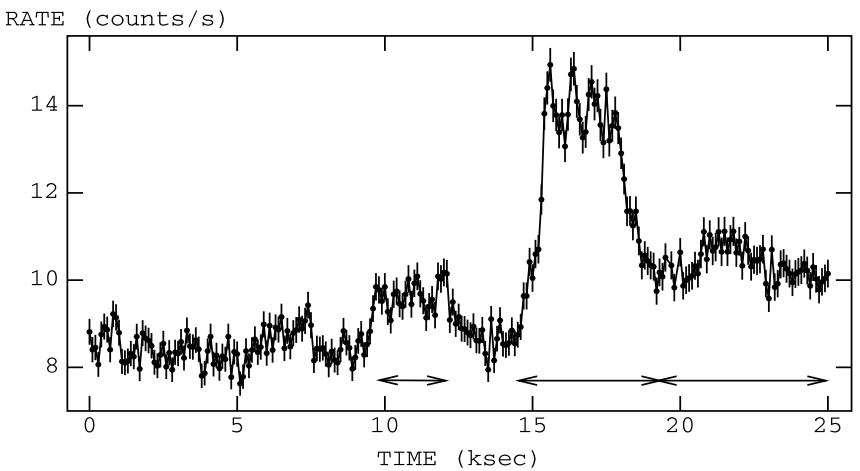

$\mathrm{AD}$ Leo

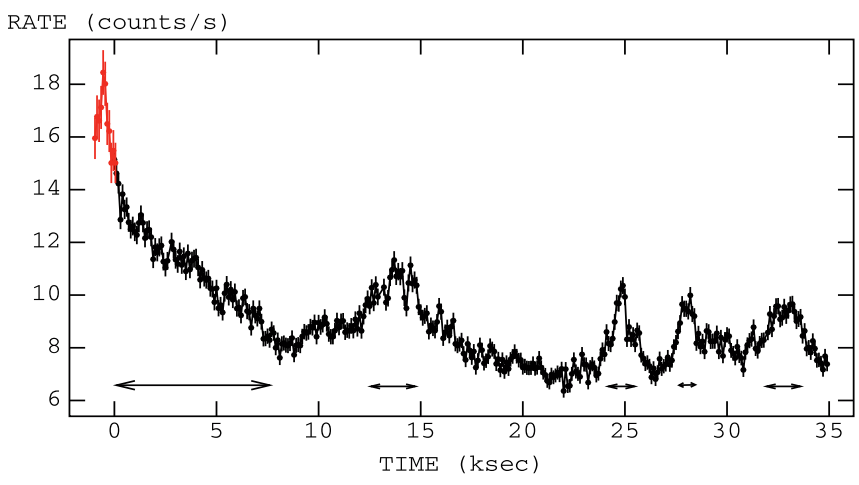

EV Lac

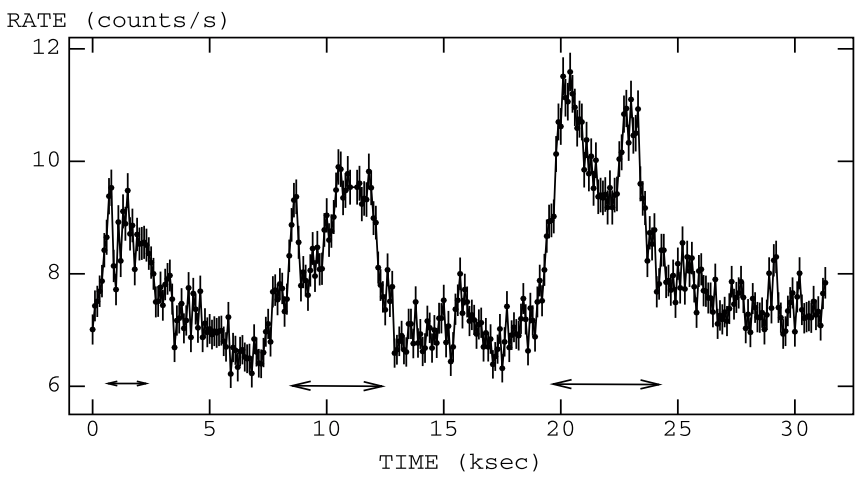

Fig. 1. Light curves of the binaries EQ Peg and AT Mic and the single stars AD Leo and EV Lac; PN (+MOS2/red) data, 100 s binning. Frequent flaring is observed on various timescales; selected flaring periods are indicated by horizontal arrows.

XMM-Newton see Güdel et al. (2004). While our observations indicate that flaring with flux increases of factors 2-3 appears
Table 3. Selection of flaring data (PN).

\begin{tabular}{lcccc}
\hline \hline & EQ Peg & AT Mic & AD Leo & EV Lac \\
\hline Median rate & 6.42 & 9.40 & 8.60 & 7.58 \\
Min. flare rate & 7.0 & 12.0 (high) & 9.5 & 8.5 \\
& & $10.0(\mathrm{med})$ & & \\
Flare intervals & $5.5-11.6$ & $14.7-19.1(\mathrm{~h})$ & $0.0-7.7$ & $0.5-2.3$ \\
(ks after & & $9.7-12.1(\mathrm{~m})$ & $12.2-14.9$ & $8.4-12.2$ \\
start of & & $19.2-25.0(\mathrm{~m})$ & $24.1-25.7$ & $19.4-24.0$ \\
observation) & & & $27.4-28.4$ & \\
& & & $32.0-33.8$ & \\
\hline
\end{tabular}

regularly on timescales of a few hours, these larger events are much rarer.

Weaker flaring (flux changes of around ten percent or lower) on timescales of a few minutes is visible throughout all four observations. In this phase the stars are in a state of low but permanent activity. This continual weak flaring makes it, in addition to the larger flares, difficult to determine a minimum or baseline flux level. This flux or activity level is denoted here as the quasi-quiescent level. It has been suggested that this emission can be interpreted as a superposition of a large number of even smaller flares with a power law distribution (Hudson 1991; Kashyap et al. 2002; Güdel et al. 2003).

The flare light curves exhibit a variety of shapes and often show substructure. The observed rise times are only somewhat shorter than the decay times for the larger impulsive flares during the AT Mic and the EV Lac observation, pointing to relatively compact flaring structures. The different types of flares as observed on the Sun are described in e.g. Feldman et al. (1995) and references therein. The rise times from quasi-quiescent to flare maximum are typically in the range of $1 \mathrm{ks}$ for the largest flares with the rise steepening towards maximum. The binaries are not resolved in these light curves, but all components are expected to be flare stars. For EQ Peg it was shown by Robrade et al. (2004) that both stars contribute to the flaring, with EQ Peg A being brighter than B by a factor of 3-4. The separation of AT Mic is too small to resolve the components.

Flaring involves significant amounts of energy release and matter transfer into the corona and may therefore involve changes in the physical properties of the coronal plasma, e.g. temperatures, emission measures, densities and abundances, a well known phenomenon from previous analyses of stellar flares. Therefore we introduce "flaring" and "quasi-quiescent" phases for each star; an interval is selected as flaring if the count rate of the time bins exceed the median count rate and the peak count rate within the interval exceeds a "minimum flare value", chosen to separate clear flare events and flickering. The selection of two phases of activity is of course somewhat arbitrary, and observations with stronger flares may be separated into three or more phases of in- or decreasing activity. With our data this was only done for the AT Mic observation, which is separated in three states of activity, the quasi-quiescent phase (QQ), the medium activity phase and the outstanding large flare. The count rates, selection criteria and selected flaring periods are summarized in Table 3. 


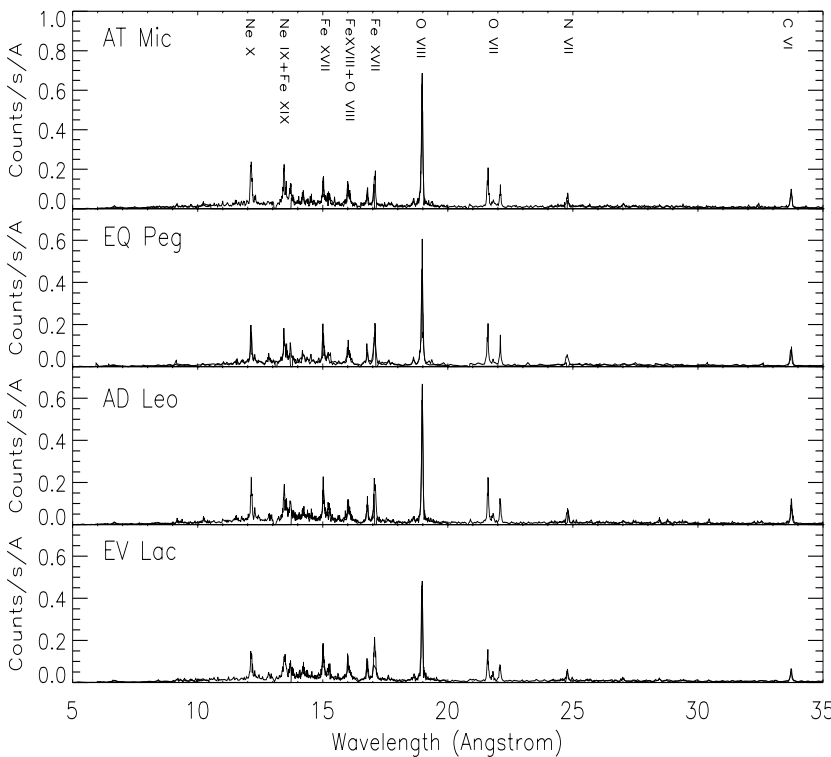

Fig. 2. RGS spectra of the sample stars, the most prominent emission features are labelled.

\subsection{Spectral analysis}

\subsubsection{RGS and EPIC spectra}

The high resolution RGS spectra of our sources in the wavelength range $5-35 \AA$ are shown in Fig. 2. In this energy range the X-ray spectra of our $\mathrm{M}$ dwarfs are remarkably similar, all being strongly dominated by emission lines. The positions of the most prominent emission features are labelled; i.e., the H-like lines of $\mathrm{CVI}, \mathrm{NVII}, \mathrm{O}$ VIII and $\mathrm{NeX}$, the strong He-like triplets of $\mathrm{O}$ VII and Ne IX as well as a number of Fe XVII - Fe XIX lines. The peak formation temperatures of these lines cover temperatures from $\sim 1.5 \mathrm{MK}(\mathrm{C} \mathrm{VI})$ over $\sim 3 \mathrm{MK}$ (O VIII) and $\sim 6 \mathrm{MK}$ (Ne X) up to $\sim 8 \mathrm{MK}$ (Fe XIX) but plasma with temperatures up to $30 \mathrm{MK}$ can contribute to these lines.

The EPIC PN data, with moderate spectral resolution, are displayed in Fig. 3 for our target stars; note that the EQ Peg EPIC spectra were extracted from an annulus because of pile up. In addition to the features mentioned in the RGS section the continuum at high energies and lines due to highly ionized iron (up to Fe XXV at $6.7 \mathrm{keV}$ ) and $\mathrm{H}$-like/He-like magnesium, silicon and sulfur are detectable. The observed features cover roughly the temperature range from 2 to $70 \mathrm{MK}$ in terms of peak formation temperatures. Inspection of the PN spectra of the sample M dwarfs shows differences in the high energy slopes, the obviously shallower slope of the AT Mic spectrum indicates the higher coronal temperatures of this source.

\subsubsection{Spectral fits and coronal temperatures}

Closer inspection of the M-dwarf spectra and their individual lines reveals further differences. To quantify these differences the spectra from each type of detector were fitted separately with the 3-T model and the 6-T model. Integration times of the spectra may differ due to varying exposure times and the influence of high background periods on the individual

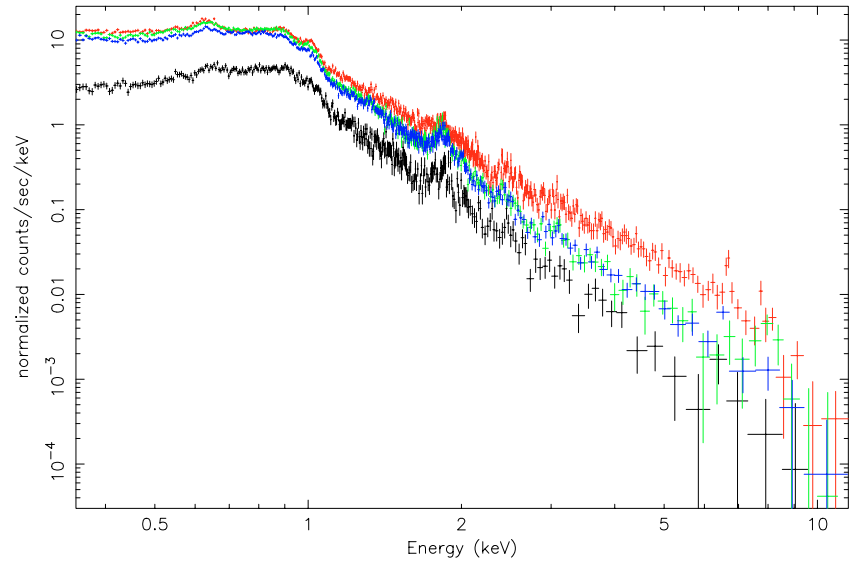

Fig. 3. PN spectra of the analysed M dwarfs; top to bottom: AT Mic (red), AD Leo (green), EV Lac (blue), EQ Peg (black). (Colour figure in electronic version.)

detectors. We examined the influence of flaring on the temperatures, emission measures and abundances of the plasma. For this purpose we extracted spectra from flaring and quasiquiescent intervals as defined in Table 3 and modelled the PN spectra for the different phases of activity, keeping all model parameters as free parameters. The results of this fitting procedure are summarized in Table 4; all errors denote $90 \%$ confidence range and were calculated by allowing variations of all other free model parameters. Note the corrected flux for EQ Peg compared to Robrade et al. (2004). The quality of these fits in terms of the $\chi^{2}$ test statistic did not improve when further components were added to the model. We point out that the calculated errors are statistical errors only and additional uncertainties are introduced by the atomic data and instrumental calibration.

Comparing the fit results for the different instruments one finds good agreement in general, but deviations of particular results are obvious. One source of uncertainty is based on the interdependence of emission measure (EM) and elemental abundances; a higher EM of a temperature component leads to the reduction of the abundance of elements producing the strongest lines in this temperature range and vice versa. The difference in fit quality between two models is often only marginal. This is especially true for EPIC data, where the best-fitting models result sometimes in higher EM with lower abundances compared to RGS data. However, a combination of these properties is in good agreement, e.g. a simple scaling of the summed EM with the summed abundances of iron and oxygen (see Table 5). Iron and oxygen are the major contributing elements of the emission of $\mathrm{M}$ dwarfs in the considered energy range; their abundances can therefore be determined best. Similar results were obtained when using the oxygen abundance for the cooler components and iron for the hotter components. Also the emission measure is in reality a continuous distribution which results in an additional interdependence between temperatures and strength of the respective EM components, noticeably in larger, often dependent, errors affecting neighbouring components. The $3-\mathrm{T}$ and the 6-T model lead to fully-consistent results on abundances for all targets; differences are within the errors and 
Table 4. Results on temperatures $(\mathrm{keV})$, emission measures $\left(10^{51} \mathrm{~cm}^{-3}\right)$ and abundances relative to solar photospheric values, errors are $90 \%$ conf. range. (d.o.f. given in brackets). $L_{\mathrm{X}}\left(10^{28} \mathrm{erg} \mathrm{s}^{-1}\right)$ indicates the $0.2-10.0 \mathrm{keV}$ luminosity. The 6-T model grid is $0.2,0.3,0.6,1.2,2.4,4.8 \mathrm{keV}$ which corresponds to $2.3,3.5,7.0,14.0,28.0,56.0 \mathrm{MK}$.

\begin{tabular}{|c|c|c|c|c|c|c|c|c|c|c|}
\hline Par. & RGS & MOS & $\mathrm{PN}$ & PN (low) & PN (high) & RGS & MOS & $\mathrm{PN}$ & PN (low) & PN (high) \\
\hline & \multicolumn{5}{|c|}{ EQ Peg - 3-T model } & \multicolumn{5}{|c|}{ EQ Peg - 6-T model } \\
\hline $\mathrm{C}$ & $0.71_{-0.14}^{+0.17}$ & - & - & - & - & $0.70_{-0.13}^{+0.18}$ & - & - & - & - \\
\hline $\mathrm{N}$ & $0.69_{-0.16}^{+0.19}$ & - & - & - & - & $0.68_{-0.15}^{+0.22}$ & - & - & - & - \\
\hline $\mathrm{O}$ & $0.54_{-0.08}^{+0.09}$ & $0.37_{-0.04}^{+0.05}$ & $0.58_{-0.08}^{+0.09}$ & $0.70_{-0.14}^{+0.18}$ & $0.48_{-0.07}^{+0.10}$ & $0.59_{-0.06}^{+0.11}$ & $0.37_{-0.05}^{+0.06}$ & $0.58_{-0.08}^{+0.09}$ & $0.73_{-0.15}^{+0.18}$ & $0.48_{-0.07}^{+0.08}$ \\
\hline $\mathrm{Ne}$ & $0.96_{-0.13}^{+0.16}$ & $0.73_{-0.12}^{+0.14}$ & $0.93_{-0.18}^{+0.19}$ & $0.92_{-0.37}^{+0.32}$ & $1.01_{-0.22}^{+0.27}$ & $0.96_{-0.13}^{+0.15}$ & $0.69_{-0.109}^{+0.09}$ & $1.00_{-0.13}^{+0.16}$ & $0.96_{-0.21}^{+0.26}$ & $1.11_{-0.17}^{+0.20}$ \\
\hline $\mathrm{Mg}$ & $0.30_{-0.14}^{+0.14}$ & $0.32_{-0.07}^{+0.08}$ & $0.25_{-0.09}^{+0.11}$ & $0.22_{-0.15}^{+0.19}$ & $0.27_{-0.11}^{+0.15}$ & $0.27_{-0.13}^{+0.15}$ & $0.33_{-0.08}^{+0.08}$ & $0.28_{-0.09}^{+0.11}$ & $0.22_{-0.14}^{+0.17}$ & $0.33_{-0.12}^{+0.14}$ \\
\hline $\mathrm{Si}$ & $0.58_{-0.29}^{+0.31}$ & $0.58_{-0.09}^{+0.10}$ & $0.40_{-0.10}^{+0.12}$ & $0.55_{-0.18}^{+0.23}$ & $0.29_{-0.11}^{+0.15}$ & $0.56_{-0.28}^{+0.34}$ & $0.60_{-0.11}^{+0.10}$ & $0.45_{-0.11}^{+0.13}$ & $0.58_{-0.19}^{+0.23}$ & $0.37_{-0.13}^{+0.15}$ \\
\hline S & $0.27_{-0.15}^{+0.18}$ & $0.68_{-0.16}^{+0.17}$ & $0.27_{-0.20}^{+0.21}$ & $0.63_{-0.34}^{+0.40}$ & $0.00_{-0.00}^{+0.18}$ & $0.28_{-0.17}^{+0.23}$ & $0.69_{-0.16}^{+0.18}$ & $0.27_{-0.22}^{+0.24}$ & $0.69_{-0.37}^{+0.44}$ & $0.00_{-0.00}^{+0.17}$ \\
\hline $\mathrm{Fe}$ & $0.46_{-0.06}^{+0.08}$ & $0.31_{-0.04}^{+0.05}$ & $0.47_{-0.07}^{+0.08}$ & $0.54_{-0.12}^{+0.16}$ & $0.43_{-0.07}^{+0.11}$ & $0.43_{-0.04}^{+0.08}$ & $0.32_{-0.05}^{+0.05}$ & $0.46_{-0.06}^{+0.08}$ & $0.52_{-0.11}^{+0.13}$ & $0.45_{-0.08}^{+0.09}$ \\
\hline kT1 & $0.26_{-0.01}^{+0.01}$ & $0.29_{-0.02}^{+0.03}$ & $0.26_{-0.01}^{+0.01}$ & $0.27_{-0.02}^{+0.04}$ & $0.24_{-0.02}^{+0.02}$ & - & - & - & - & - \\
\hline EM1 & $1.62_{-0.28}^{+0.32}$ & $2.81_{-0.53}^{+0.81}$ & $1.81_{-0.39}^{+0.41}$ & $1.63_{-0.48}^{+0.81}$ & $2.06_{-0.54}^{+0.56}$ & $0.52_{-0.15}^{+0.15}$ & $0.17_{-0.12}^{+0.37}$ & $0.56_{-0.20}^{+0.21}$ & $0.30_{-0.23}^{+0.22}$ & $0.96_{-0.36}^{+0.37}$ \\
\hline kT2 & $0.63_{-0.02}^{+0.02}$ & $0.60_{-0.02}^{+0.03}$ & $0.64_{-0.02}^{+0.02}$ & $0.64_{-0.04}^{+0.10}$ & $0.64_{-0.03}^{+0.03}$ & - & - & - & - & - \\
\hline EM2 & $2.18_{-0.31}^{+0.36}$ & $3.26_{-0.66}^{+0.63}$ & $2.57_{-0.48}^{+0.54}$ & $1.70_{-0.70}^{+0.68}$ & $3.33_{-0.82}^{+0.80}$ & $0.99_{-0.29}^{+0.26}$ & $2.88_{-0.69}^{+0.63}$ & $1.37_{-0.46}^{+0.51}$ & $1.31_{-0.59}^{+0.62}$ & $1.37_{-0.65}^{+0.70}$ \\
\hline kT3 & $1.20_{-0.15}^{+0.14}$ & $1.53_{-0.12}^{+0.16}$ & $1.43_{-0.12}^{+0.14}$ & $1.35_{-0.15}^{+0.13}$ & $1.64_{-0.21}^{+0.28}$ & - & - & - & - & - \\
\hline EM3 & $0.96_{-0.19}^{+0.18}$ & $1.02_{-0.18}^{+0.17}$ & $0.99_{-0.18}^{+0.17}$ & $0.94_{-0.22}^{+0.23}$ & $1.01_{-0.28}^{+0.28}$ & $2.14_{-0.34}^{+0.29}$ & $2.82_{-0.45}^{+0.50}$ & $2.31_{-0.45}^{+0.49}$ & $1.70_{-0.49}^{+0.64}$ & $2.74_{-0.64}^{+0.78}$ \\
\hline EM4 & - & - & - & - & - & $1.14_{-0.20}^{+0.15}$ & $0.92_{-0.29}^{+0.23}$ & $0.90_{-0.20}^{+0.21}$ & $0.92_{-0.22}^{+0.25}$ & $0.77_{-0.39}^{+0.32}$ \\
\hline EM5 & - & - & - & - & - & $0.00_{-0.00}^{+0.20}$ & $0.27_{-0.22}^{+0.10}$ & $0.23_{-0.15}^{+0.11}$ & $0.11_{-0.11}^{+0.13}$ & $0.44_{-0.24}^{+0.18}$ \\
\hline EM6 & - & - & - & - & - & $0.00_{-0.00}^{+0.22}$ & $0.00_{-0.00}^{+0.10}$ & $0.00_{-0.00}^{+0.04}$ & $0.00_{-0.00}^{+0.07}$ & $0.00_{-0.00}^{+0.10}$ \\
\hline red. $\chi^{2}$ & $1.26(731)$ & $0.99(291)$ & $1.17(365)$ & $0.97(287)$ & $1.06(303)$ & $1.29(731)$ & 0.99 (291) & $1.22(365)$ & $1.00(287)$ & $1.10(303)$ \\
\hline \multirow[t]{2}{*}{$L_{\mathrm{X}}$} & 5.83 & 7.25 & 6.59 & 5.87 & 7.30 & 5.86 & 7.27 & 6.61 & 5.88 & 7.33 \\
\hline & \multicolumn{5}{|c|}{ AT Mic } & & & & & \\
\hline $\mathrm{C}$ & $0.80_{-0.11}^{+0.13}$ & - & - & - & - & $0.79_{-0.11}^{+0.13}$ & - & - & - & - \\
\hline $\mathrm{N}$ & $0.65_{-0.11}^{+0.13}$ & - & - & - & - & $0.66_{-0.11}^{+0.13}$ & - & - & - & - \\
\hline $\mathrm{O}$ & $0.58_{-0.06}^{+0.07}$ & $0.37_{-0.03}^{+0.04}$ & $0.45_{-0.04}^{+0.06}$ & $0.44_{-0.04}^{+0.05}$ & $0.46_{-0.07}^{+0.09}$ & $0.62_{-0.06}^{+0.08}$ & $0.37_{-0.03}^{+0.04}$ & $0.43_{-0.03}^{+0.03}$ & $0.43_{-0.04}^{+0.05}$ & $0.45_{-0.06}^{+0.06}$ \\
\hline $\mathrm{Ne}$ & $1.31_{-0.14}^{+0.16}$ & $0.83_{-0.10}^{+0.08}$ & $0.67_{-0.09}^{+0.07}$ & $0.63_{-0.08}^{+0.09}$ & $0.72_{-0.16}^{+0.24}$ & $1.26_{-0.14}^{+0.15}$ & $0.85_{-0.06}^{+0.08}$ & $0.78_{-0.05}^{+0.05}$ & $0.73_{-0.07}^{+0.08}$ & $0.74_{-0.11}^{+0.13}$ \\
\hline $\mathrm{Mg}$ & $0.30_{-0.09}^{+0.10}$ & $0.24_{-0.05}^{+0.05}$ & $0.14_{-0.04}^{+0.04}$ & $0.14_{-0.05}^{+0.06}$ & $0.13_{-0.08}^{+0.09}$ & $0.27_{-0.09}^{+0.09}$ & $0.28_{-0.06}^{+0.06}$ & $0.20_{-0.04}^{+0.04}$ & $0.20_{-0.06}^{+0.05}$ & $0.20_{-0.09}^{+0.10}$ \\
\hline $\mathrm{Si}$ & $0.40_{-0.19}^{+0.19}$ & $0.49_{-0.08}^{+0.09}$ & $0.25_{-0.04}^{+0.05}$ & $0.28_{-0.06}^{+0.07}$ & $0.21_{-0.09}^{+0.10}$ & $0.39_{-0.19}^{+0.19}$ & $0.47_{-0.07}^{+0.08}$ & $0.31_{-0.05}^{+0.05}$ & $0.37_{-0.07}^{+0.07}$ & $0.28_{-0.10}^{+0.11}$ \\
\hline S & $0.17_{-0.10}^{+0.11}$ & $0.44_{-0.14}^{+0.14}$ & $0.27_{-0.11}^{+0.11}$ & $0.27_{-0.14}^{+0.15}$ & $0.11_{-0.11}^{+0.24}$ & $0.18_{-0.11}^{+0.12}$ & $0.50_{-0.13}^{+0.14}$ & $0.22_{-0.10}^{+0.11}$ & $0.25_{-0.15}^{+0.18}$ & $0.16_{-0.16}^{+0.20}$ \\
\hline $\mathrm{Fe}$ & $0.35_{-0.04}^{+0.05}$ & $0.18_{-0.02}^{+0.03}$ & $0.21_{-0.02}^{+0.02}$ & $0.23_{-0.08}^{+0.07}$ & $0.20_{-0.03}^{+0.06}$ & $0.32_{-0.04}^{+0.04}$ & $0.23_{-0.03}^{+0.03}$ & $0.23_{-0.02}^{+0.02}$ & $0.24_{-0.03}^{+0.03}$ & $0.27_{-0.04}^{+0.06}$ \\
\hline kT1 & $0.27_{-0.01}^{+0.01}$ & $0.36_{-0.03}^{+0.04}$ & $0.25_{-0.01}^{+0.01}$ & $0.26_{-0.01}^{+0.01}$ & $0.24_{-0.03}^{+0.02}$ & - & - & - & - & - \\
\hline EM1 & $4.97_{-0.69}^{+0.79}$ & $11.20_{-2.39}^{+5.64}$ & $7.42_{-1.60}^{+1.01}$ & $8.20_{-1.23}^{+1.33}$ & $6.50_{-3.23}^{+2.30}$ & $0.73_{-0.26}^{+0.31}$ & $0.00_{-0.00}^{+0.23}$ & $2.61_{-0.49}^{+0.54}$ & $2.40_{-0.65}^{+0.75}$ & $3.06_{-1.10}^{+1.11}$ \\
\hline kT2 & $0.63_{-0.01}^{+0.02}$ & $0.60_{-0.04}^{+0.18}$ & $0.64_{-0.02}^{+0.02}$ & $0.65_{-0.02}^{+0.02}$ & $0.62_{-0.03}^{+0.03}$ & - & - & - & - & - \\
\hline EM2 & $7.34_{-0.93}^{+1.01}$ & $9.19_{-5.42}^{+3.26}$ & $16.01_{-1.49}^{+2.46}$ & $13.91_{-1.81}^{+1.66}$ & $19.15_{-6.17}^{+4.75}$ & $4.06_{-0.78}^{+0.80}$ & $8.46_{-1.07}^{+1.6}$ & $6.58_{-1.11}^{+0.96}$ & $6.92_{-1.81}^{+1.66}$ & $6.72_{-2.56}^{+2.75}$ \\
\hline kT3 & $1.98_{-0.28}^{+0.39}$ & $2.06_{-0.12}^{+0.42}$ & $2.56_{-0.19}^{+0.48}$ & $2.04_{-1.84}^{+1.34}$ & $3.00_{-0.03}^{+0.11}$ & - & - & - & - & - \\
\hline EM3 & $6.55_{-0.69}^{-0.66}$ & $7.93_{-1.08}^{+0.12}$ & $5.52_{-1.09}^{+0.19}$ & $2.39_{-0.59}^{+0.04}$ & $11.86_{-2.18}^{+2.78}$ & $7.28_{-0.96}^{+1.12}$ & $10.16_{-1.68}^{+1.94}$ & $12.71_{-0.84}^{+1.60}$ & $11.51_{-1.33}^{+2.15}$ & $11.12_{-2.93}^{+3.19}$ \\
\hline EM4 & - & - & - & - & - & $1.78_{-1.15}^{+1.18}$ & $4.57_{-1.79}^{+1.13}$ & $0.84_{-0.84}^{+1.00}$ & $1.49_{-0.72}^{+0.72}$ & $4.98_{-2.14}^{+2.11}$ \\
\hline EM5 & - & - & - & - & - & $4.02_{-4.02}^{+2.39}$ & $2.24_{-1.28}^{+2.16}$ & $5.74_{-0.59}^{+0.99}$ & $1.85_{-0.32}^{+0.42}$ & $6.27_{-3.11}^{+3.19}$ \\
\hline EM6 & - & - & - & - & - & $2.39_{-2.39}^{+3.17}$ & $1.62_{-0.99}^{+0.60}$ & $0.21_{-0.21}^{+0.47}$ & $0.00_{-0.00}^{+0.09}$ & $4.02_{-1.48}^{+1.44}$ \\
\hline red. $\chi^{2}$ & $1.15(1299)$ & $1.39(231)$ & $1.31(537)$ & $1.14(424)$ & $1.10(511)$ & $1.16(1299)$ & $1.38(231)$ & $1.34(537)$ & $1.23(424)$ & $1.06(511)$ \\
\hline$L_{\mathrm{X}}$ & 22.71 & 27.64 & 28.95 & 23.61 & 39.65 & 23.49 & 27.87 & 29.01 & 23.68 & 39.75 \\
\hline
\end{tabular}

negligible when compared to the uncertainties arising from the use of different detectors.

One effect of calibration uncertainties is that the best fit models of the different detectors do vary in X-ray flux, especially below $1.0 \mathrm{keV}$. Typical RGS models result in a $\sim 20 \%$ lower X-ray flux, mainly in the energy range $0.2-1.0 \mathrm{keV}$ compared to EPIC, whereas the PN models are a few percent more luminous than the MOS models. The RGS luminosities in Table 4 have been extrapolated to the $0.2-10.0 \mathrm{keV}$ band for comparison with the EPIC luminosities. Smaller 
Table 4. continued.

\begin{tabular}{|c|c|c|c|c|c|c|c|c|c|c|}
\hline Param. & RGS & MOS & PN & PN (low) & PN (high) & RGS & MOS & PN & PN (low) & PN (high) \\
\hline \multicolumn{11}{|c|}{ AD Leo } \\
\hline $\mathrm{C}$ & $0.83_{-0.09}^{+0.10}$ & - & - & - & - & $0.85_{-0.10}^{+0.11}$ & - & - & - & - \\
\hline $\mathrm{N}$ & $0.73_{-0.10}^{+0.11}$ & - & - & - & - & $0.76_{-0.10}^{+0.12}$ & - & - & - & - \\
\hline $\mathrm{O}$ & $0.52_{-0.05}^{+0.05}$ & $0.41_{-0.03}^{+0.04}$ & $0.45_{-0.03}^{+0.04}$ & $0.44_{-0.05}^{+0.06}$ & $0.44_{-0.04}^{+0.06}$ & $0.58_{-0.05}^{+0.06}$ & $0.41_{-0.03}^{+0.04}$ & $0.46_{-0.04}^{+0.04}$ & $0.47_{-0.06}^{+0.06}$ & $0.44_{-0.04}^{+0.05}$ \\
\hline $\mathrm{Ne}$ & $1.08_{-0.10}^{+0.11}$ & $0.86_{-0.08}^{+0.11}$ & $0.68_{-0.07}^{+0.07}$ & $0.68_{-0.11}^{+0.10}$ & $0.71_{-0.10}^{+0.10}$ & $1.07_{-0.10}^{+0.12}$ & $0.83_{-0.05}^{+0.06}$ & $0.74_{-0.06}^{+0.06}$ & $0.71_{-0.08}^{+0.10}$ & $0.75_{-0.07}^{+0.08}$ \\
\hline $\mathrm{Mg}$ & $0.44_{-0.09}^{+0.09}$ & $0.30_{-0.05}^{+0.06}$ & $0.14_{-0.04}^{+0.04}$ & $0.14_{-0.05}^{+0.05}$ & $0.13_{-0.05}^{+0.06}$ & $0.41_{-0.08}^{+0.08}$ & $0.30_{-0.05}^{+0.05}$ & $0.17_{-0.04}^{+0.04}$ & $0.15_{-0.04}^{+0.06}$ & $0.17_{-0.05}^{+0.07}$ \\
\hline $\mathrm{Si}$ & $0.56_{-0.16}^{+0.17}$ & $0.67_{-0.07}^{+0.08}$ & $0.40_{-0.05}^{+0.06}$ & $0.40_{-0.08}^{+0.08}$ & $0.41_{-0.08}^{+0.09}$ & $0.56_{-0.17}^{+0.17}$ & $0.67_{-0.07}^{+0.07}$ & $0.45_{-0.06}^{+0.06}$ & $0.42_{-0.07}^{+0.09}$ & $0.46_{-0.07}^{+0.09}$ \\
\hline $\mathrm{S}$ & $0.41_{-0.09}^{+0.10}$ & $0.61_{-0.12}^{+0.11}$ & $0.36_{-0.12}^{+0.12}$ & $0.40_{-0.18}^{+0.21}$ & $0.26_{-0.13}^{+0.17}$ & $0.46_{-0.11}^{+0.12}$ & $0.63_{-0.12}^{+0.12}$ & $0.36_{-0.13}^{+0.14}$ & $0.43_{-0.20}^{+0.22}$ & $0.26_{-0.14}^{+0.17}$ \\
\hline $\mathrm{Fe}$ & $0.47_{-0.04}^{+0.04}$ & $0.30_{-0.03}^{+0.03}$ & $0.27_{-0.02}^{+0.03}$ & $0.29_{-0.03}^{+0.03}$ & $0.27_{-0.03}^{+0.03}$ & $0.44_{-0.04}^{+0.05}$ & $0.31_{-0.02}^{+0.03}$ & $0.28_{-0.02}^{+0.02}$ & $0.29_{-0.03}^{+0.03}$ & $0.29_{-0.03}^{+0.04}$ \\
\hline $\mathrm{kT} 1$ & $0.26_{-0.01}^{+0.01}$ & $0.31_{-0.03}^{+0.02}$ & $0.24_{-0.01}^{+0.01}$ & $0.24_{-0.01}^{+0.01}$ & $0.25_{-0.01}^{+0.01}$ & - & - & - & - & - \\
\hline EM1 & $1.23_{-0.14}^{+0.15}$ & $1.82_{-0.32}^{+0.28}$ & $1.63_{-0.21}^{+0.21}$ & $1.75_{-0.30}^{+0.27}$ & $1.69_{-0.39}^{+0.29}$ & $0.29_{-0.10}^{+0.12}$ & $0.00_{-0.00}^{+0.02}$ & $0.78_{-0.11}^{+0.11}$ & $0.83_{-0.14}^{+0.14}$ & $0.71_{-0.17}^{+0.16}$ \\
\hline $\mathrm{kT} 2$ & $0.63_{-0.02}^{+0.02}$ & $0.61_{-0.02}^{+0.02}$ & $0.62_{-0.01}^{+0.01}$ & $0.60_{-0.02}^{+0.02}$ & $0.62_{-0.02}^{+0.02}$ & - & - & - & - & - \\
\hline EM2 & $1.41_{-0.13}^{+0.15}$ & $2.06_{-0.29}^{+0.27}$ & $3.22_{-0.29}^{+0.30}$ & $2.66_{-0.27}^{+0.35}$ & $3.71_{-0.48}^{+0.62}$ & $0.85_{-0.13}^{+0.13}$ & $1.73_{-0.19}^{+0.15}$ & $0.98_{-0.23}^{+0.29}$ & $1.00_{-0.27}^{+0.35}$ & $1.19_{-0.36}^{+0.46}$ \\
\hline kT3 & $1.33_{-0.10}^{+0.15}$ & $1.54_{-0.08}^{+0.09}$ & $2.18_{-0.29}^{+0.50}$ & $2.65_{-0.64}^{+2.64}$ & $1.96_{-0.23}^{+0.50}$ & - & - & - & - & - \\
\hline EM3 & $0.67_{-0.09}^{+0.08}$ & $0.82_{-0.09}^{+0.08}$ & $0.43_{-0.10}^{+0.10}$ & $0.22_{-0.010}^{+0.10}$ & $0.77_{-0.23}^{+0.17}$ & $1.40_{-0.14}^{+0.17}$ & $2.02_{-0.24}^{+0.26}$ & $2.97_{-0.38}^{+0.18}$ & $2.50_{-0.31}^{+0.30}$ & $3.19_{-0.55}^{+0.36}$ \\
\hline EM4 & - & - & - & - & - & $0.64_{-0.16}^{+0.12}$ & $0.69_{-0.13}^{+0.12}$ & $0.08_{-0.08}^{+0.21}$ & $0.00_{-0.00}^{+0.22}$ & $0.46_{-0.21}^{+0.30}$ \\
\hline EM5 & - & - & - & - & - & $0.00_{-0.00}^{+0.27}$ & $0.23_{-0.09}^{+0.05}$ & $0.41_{-0.13}^{+0.06}$ & $0.21_{-0.15}^{+0.10}$ & $0.51_{-0.29}^{+0.09}$ \\
\hline EM6 & - & - & - & - & - & $0.09_{-0.09}^{+0.16}$ & $0.00_{-0.00}^{+0.04}$ & $0.00_{-0.00}^{+0.04}$ & $0.00_{-0.00}^{+0.09}$ & $0.00_{-0.00}^{+0.12}$ \\
\hline red. $\chi^{2}$ & $1.29(1444)$ & $1.31(206)$ & $1.27(442)$ & $1.13(395)$ & $1.15(435)$ & $1.30(1444)$ & $1.31(206)$ & $1.29(442)$ & $1.12(395)$ & $1.17(435)$ \\
\hline \multirow[t]{2}{*}{$L_{\mathrm{X}}$} & 4.12 & 4.96 & 5.39 & 4.71 & 6.28 & 4.13 & 4.97 & 5.41 & 4.71 & 6.30 \\
\hline & \multicolumn{10}{|c|}{ EV Lac } \\
\hline $\mathrm{C}$ & $0.51_{-0.07}^{+0.08}$ & - & - & - & - & $0.54_{-0.07}^{+0.08}$ & - & - & - & - \\
\hline $\mathrm{N}$ & $0.45_{-0.08}^{+0.08}$ & - & - & - & - & $0.47_{-0.08}^{+0.09}$ & - & - & - & - \\
\hline $\mathrm{O}$ & $0.39_{-0.04}^{+0.05}$ & $0.31_{-0.03}^{+0.03}$ & $0.34_{-0.03}^{+0.03}$ & $0.35_{-0.03}^{+0.04}$ & $0.32_{-0.04}^{+0.06}$ & $0.45_{-0.04}^{+0.05}$ & $0.31_{-0.03}^{+0.03}$ & $0.35_{-0.02}^{+0.03}$ & $0.36_{-0.03}^{+0.04}$ & $0.34_{-0.04}^{+0.04}$ \\
\hline $\mathrm{Ne}$ & $0.69_{-0.08}^{+0.09}$ & $0.56_{-0.08}^{+0.09}$ & $0.48_{-0.05}^{+0.05}$ & $0.50_{-0.06}^{+0.06}$ & $0.49_{-0.10}^{+0.09}$ & $0.68_{-0.08}^{+0.08}$ & $0.62_{-0.05}^{+0.06}$ & $0.51_{-0.03}^{+0.04}$ & $0.52_{-0.05}^{+0.05}$ & $0.49_{-0.06}^{+0.06}$ \\
\hline $\mathrm{Mg}$ & $0.35_{-0.08}^{+0.08}$ & $0.26_{-0.05}^{+0.06}$ & $0.16_{-0.03}^{+0.03}$ & $0.20_{-0.04}^{+0.04}$ & $0.12_{-0.05}^{+0.06}$ & $0.31_{-0.07}^{+0.08}$ & $0.29_{-0.05}^{+0.05}$ & $0.19_{-0.02}^{+0.03}$ & $0.22_{-0.04}^{+0.04}$ & $0.13_{-0.04}^{+0.06}$ \\
\hline $\mathrm{Si}$ & $0.63_{-0.17}^{+0.17}$ & $0.49_{-0.07}^{+0.08}$ & $0.36_{-0.04}^{+0.05}$ & $0.42_{-0.06}^{+0.06}$ & $0.30_{-0.07}^{+0.07}$ & $0.62_{-0.17}^{+0.17}$ & $0.52_{-0.07}^{+0.07}$ & $0.40_{-0.04}^{+0.04}$ & $0.45_{-0.06}^{+0.06}$ & $0.32_{-0.05}^{+0.08}$ \\
\hline $\mathrm{S}$ & $0.18_{-0.08}^{+0.09}$ & $0.48_{-0.12}^{+0.13}$ & $0.32_{-0.09}^{+0.10}$ & $0.40_{-0.13}^{+0.13}$ & $0.18_{-0.13}^{+0.14}$ & $0.21_{-0.09}^{+0.10}$ & $0.51_{-0.12}^{+0.13}$ & $0.33_{-0.09}^{+0.10}$ & $0.41_{-0.12}^{+0.15}$ & $0.19_{-0.12}^{+0.15}$ \\
\hline $\mathrm{Fe}$ & $0.38_{-0.04}^{+0.04}$ & $0.24_{-0.03}^{+0.03}$ & $0.24_{-0.02}^{+0.02}$ & $0.27_{-0.02}^{+0.02}$ & $0.22_{-0.03}^{+0.03}$ & $0.35_{-0.03}^{+0.04}$ & $0.26_{-0.03}^{+0.03}$ & $0.26_{-0.02}^{+0.02}$ & $0.28_{-0.03}^{+0.02}$ & $0.23_{-0.02}^{+0.04}$ \\
\hline kT1 & $0.27_{-0.01}^{+0.01}$ & $0.33_{-0.03}^{+0.04}$ & $0.25_{-0.02}^{+0.02}$ & $0.26_{-0.01}^{+0.01}$ & $0.25_{-0.02}^{+0.01}$ & - & - & - & - & - \\
\hline EM1 & $1.42_{-0.21}^{+0.27}$ & $2.41_{-0.48}^{+0.95}$ & $2.01_{-0.24}^{+0.27}$ & $2.07_{-0.27}^{+0.29}$ & $2.06_{-0.69}^{+0.51}$ & $0.23_{-0.08}^{+0.08}$ & $0.00_{-0.00}^{+0.09}$ & $0.72_{-0.12}^{+0.12}$ & $0.63_{-0.13}^{+0.13}$ & $0.92_{-0.24}^{+0.23}$ \\
\hline $\mathrm{kT} 2$ & $0.63_{-0.01}^{+0.02}$ & $0.64_{-0.03}^{+0.07}$ & $0.61_{-0.01}^{+0.01}$ & $0.61_{-0.01}^{+0.01}$ & $0.61_{-0.02}^{+0.02}$ & - & - & - & - & - \\
\hline EM2 & $2.04_{-0.25}^{+0.24}$ & $2.74_{-0.87}^{+0.46}$ & $3.62_{-0.34}^{+0.30}$ & $3.10_{-0.31}^{+0.31}$ & $4.41_{-0.68}^{+0.95}$ & $0.99_{-0.22}^{+0.21}$ & $2.00_{-0.27}^{+0.26}$ & $1.54_{-0.32}^{+0.30}$ & $1.69_{-0.38}^{+0.35}$ & $1.28_{-0.37}^{+0.70}$ \\
\hline kT3 & $1.62_{-0.27}^{+0.55}$ & $1.85_{-0.17}^{+0.24}$ & $2.18_{-0.23}^{+0.30}$ & $2.24_{-0.39}^{+0.74}$ & $2.05_{-0.21}^{+0.48}$ & - & - & - & - & - \\
\hline EM3 & $0.60_{-0.14}^{+0.14}$ & $0.72_{-0.13}^{+0.12}$ & $0.53_{-0.09}^{+0.11}$ & $0.28_{-0.09}^{+0.10}$ & $1.11_{-0.32}^{+0.23}$ & $2.11_{-0.23}^{+0.25}$ & $2.85_{-0.38}^{+0.44}$ & $3.22_{-0.32}^{+0.30}$ & $2.74_{-0.34}^{+0.41}$ & $4.07_{-0.82}^{+0.61}$ \\
\hline EM4 & - & - & - & - & - & $0.42_{-0.22}^{+0.17}$ & $0.45_{-0.21}^{+0.20}$ & $0.20_{-0.17}^{+0.14}$ & $0.12_{-0.12}^{+0.15}$ & $0.43_{-0.15}^{+0.43}$ \\
\hline EM5 & - & - & - & - & - & $0.00_{-0.00}^{+0.49}$ & $0.40_{-0.19}^{+0.08}$ & $0.44_{-0.10}^{+0.06}$ & $0.24_{-0.12}^{+0.06}$ & $0.81_{-0.05}^{+0.11}$ \\
\hline EM6 & - & - & - & - & - & $0.26_{-0.26}^{+0.19}$ & $0.00_{-0.00}^{+0.08}$ & $0.00_{-0.00}^{+0.04}$ & $0.00_{-0.00}^{+0.04}$ & $0.00_{-0.00}^{+0.15}$ \\
\hline red. $\chi^{2}$ & $1.11(1173)$ & 1.35 (196) & $1.32(479)$ & $1.15(459)$ & 1.19 (457) & $1.12(1173)$ & 1.40 (196) & $1.33(479)$ & $1.15(459)$ & $1.20(457)$ \\
\hline$L_{\mathrm{X}}$ & 4.26 & 5.30 & 5.53 & 4.96 & 6.66 & 4.37 & 5.31 & 5.54 & 4.97 & 6.68 \\
\hline
\end{tabular}

cross-calibration errors, particularly at energies below $0.5 \mathrm{keV}$, are also seen in observations made with the same detector but different setups, especially with different filters. Enclosed energy correction from annular extraction regions, necessary for piled-up sources, also influences the measured flux, which can be reduced by up to $\sim 10 \%$. Additional effects are expected for the X-ray flux of the binaries, but in our case they are dominated by one component and/or the separation is small 


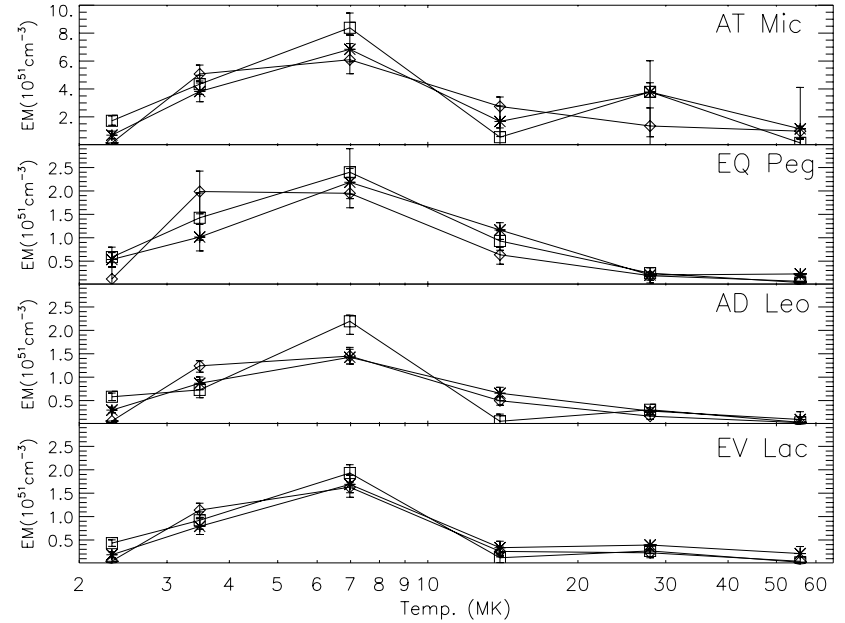

Fig. 4. EMDs as derived with the 6-T model and scaled with the summed abundance of iron and oxygen, total observation each (RGS: Asterisks, MOS: Diamonds, PN: Squares).

Table 5. Deviations of the XMM instruments from the mean scaled summed $\mathrm{EM}\left(\Sigma E M *\left(A_{\mathrm{Fe}}+A_{0}\right)\left[10^{51} \mathrm{~cm}^{-3}\right]\right)$.

\begin{tabular}{l|cccc}
\hline \hline Instr. & EQ Peg & AT Mic & AD Leo & EV Lac \\
\hline RGS & $-5 \%$ & $+3 \%$ & $-4 \%$ & $-5 \%$ \\
PN & $+10 \%$ & $+7 \%$ & $+9 \%$ & $+7 \%$ \\
MOS & $-5 \%$ & $-11 \%$ & $-4 \%$ & $-3 \%$ \\
\hline sc. $\Sigma$ EM & 4.51 & 15.32 & 3.08 & 2.97 \\
\hline
\end{tabular}

compared to the used extraction region. Calibration efforts are still ongoing, see e.g. Kirsch et al. (2004) for updated XMMNewton (cross)-calibration.

We note that that the EPIC detectors are not very appropriate to measure changes in the abundance pattern for the different phases of activity as they sometimes result in unphysical solutions like a zero abundance. On the other hand, the RGS is not very sensitive to high temperature plasma and in the 6 -T models applied to RGS data sometimes plasma is put into the hottest components, that is not seen in the EPIC models. Since the contributions from these components in the RGS are also compatible with a zero value, we expect the EPIC models to give a more realistic EMD at these energies. Overall we consider the abundance determination below $\sim 1.5 \mathrm{keV}$ to be more accurate for RGS data, while properties of the hotter plasma are best determined with EPIC data. Therefore we analysed additionally for the different phases of activity the distribution of elemental abundances with RGS data, and the emission measures with PN data using a fixed abundance pattern. The results of flare-related changes will be presented in Sect. 3.3.

The derived temperature structure is consistent among the detectors, independent of the model used, when accounting for the above mentioned uncertainties. The temperature distributions of all sample stars peak consistently around 7-8 MK $(1 \mathrm{keV} \equiv 11.6 \mathrm{MK})$ with the bulk of the plasma being located between 2-20 MK for AD Leo, EQ Peg and EV Lac, whereas the AT Mic distribution extends to somewhat higher temperatures. In the EPIC 3-T models the high temperature component tends to be hotter and/or stronger compared to RGS.

The EMDs for the total observation of our sample stars as derived from the 6-T model with the different detectors onboard XMM-Newton are shown in Fig. 4. Undetected components are plotted with their upper limits. The EMDs were scaled with the summed abundance of iron and oxygen and are in good agreement when comparing the individual instruments but individual temperature components are sometimes more pronounced. The shape of the distribution looks similar for our sample stars but the contributions of the hot plasma differ and are strongest for AT Mic, which is also the X-ray brightest source in our sample. AT Mic is associated with the $\beta$ Pictoris moving group (Barrado y Navascues et al. 1999; Zuckerman et al. 2001), with a probable age of about 10-20 Myr whereas the other stars are members of the kinematic young disk population with ages of the order of $1 \mathrm{Gyr}$. At this age AT Mic would be a still contracting pre-main sequence system, therefore the stars are larger with higher $L_{\mathrm{bol}}$ and because they are in the saturated regime, they have consequently higher $L_{\mathrm{X}}$.

We also investigated joint fits, where data of different instruments, e.g. RGS and MOS, are fitted simultaneously. While combined fits with datasets of different detectors usually turn out a weighted, intermediate result, this is not the case when taking RGS data in the full energy range and combining it with MOS data above 0.8 or $1.0 \mathrm{keV}$. This choice is reasonable because the relative energy resolution of the EPIC instruments improves towards higher energies, whereas the effective area of the RGS drops above $1.0 \mathrm{keV}$. The derived abundances in these fits increase by $\sim 20-30 \%$, whereas emission measure drops, compared to the pure RGS model; the derived temperatures change only marginally. The effect on individual elements and respectively emission measure components slightly depends on the chosen overlap and is similarly observed with PN data. Discarding the RGS data at higher energies, e.g. above $1.5 \mathrm{keV}$, has only minor influences on the derived models for our stars.

\subsubsection{Coronal abundances}

The abundance patterns of our sample stars are shown in Fig. 5, where we plot the abundances with respect to solar photospheric abundances against the FIP (First Ionization Potential) of the corresponding element. Here we show the results for the total observation that are constrained best. The data of the individual stars are separated by an offset of one on the vertical axis and a dotted line is plotted at 0.5 solar abundance to guide the eye. For elements with stronger lines in the RGS we accept these values (i.e., for $\mathrm{Fe}, \mathrm{C}, \mathrm{O}, \mathrm{N}, \mathrm{Ne}$ ), for the other elements we use the MOS values, which has a better spectral resolution compared to PN. The higher oxygen values obtained with the solar oxygen abundance given by Allende Prieto et al. (2001) are also plotted, indicating the current uncertainties in the solar abundance pattern. A discussion of problems in determining solar photospheric abundances can be found in Holweger (2001).

The determined metallicities of our sample stars vary only moderately between the individual stars; we do not consider 


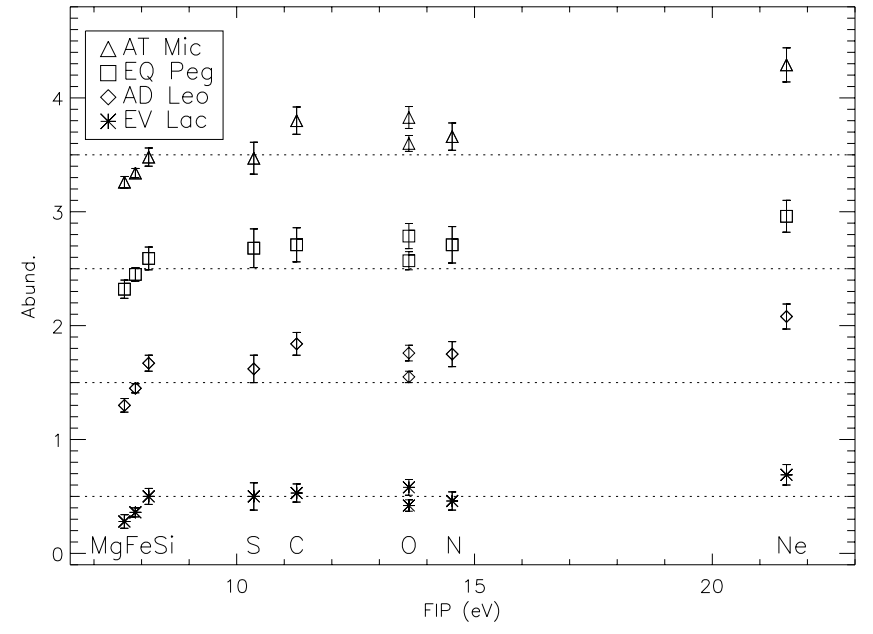

Fig. 5. Elemental abundances in stellar coronae relative to solar photospheric values vs. FIP for our sample stars; an inverse FIP effect is present in all stars. Each star is shifted by one solar abundance on the vertical axis, the dashed lines indicate 0.5 solar abundance in each case.

these differences to be very significant. However, all abundances are subsolar when compared to solar photospheric values. Unfortunately, photospheric abundances for $\mathrm{M}$ dwarfs are usually unknown and the rare measurements have large uncertainties. Naftilan et al. (1992) published photospheric abundances for AD Leo, arguing for a metallicity of $\sim 0.4$ solar for the analysed low FIP metals; iron was determined to $\sim 0.5$ solar, in agreement with our coronal measurements but chromospheric contamination could not be ruled out completely. Zboril et al. (1998) published a metallicity of $\sim 0.6$ solar for a number of $\mathrm{K}$ and $\mathrm{M}$ dwarfs from optical/IR observations. These abundance values are challenging because our sample stars are members of the kinematic young disk population (with AT Mic being even younger), that are fast rotating (Delfosse et al. 1998) and highly X-ray active, which indicates a younger age in contrast to the reduced metallicity one would associate with older stars.

If the photospheric metallicities in our sample stars are indeed as low as suggested by the authors cited above, i.e. in the range $0.4-0.6$ solar, the abundances of low FIP elements would actually be consistent with the stellar photospheric values or possibly slightly depleted, while the high FIP elements would actually be enhanced in the coronal material. An overall coronal metal abundance deficiency would then not be present, rather one would find a situation reminiscent of the solar corona, i.e., an enhancement of specific (but different) elements.

When comparing star-to-star variations of abundance as a function of FIP, one finds a tendency that the variations of low FIP elements are smaller than those of high FIP elements, e.g. neon varies by more than $50 \%$ compared to iron where the effect is below $10 \%$. Whereas the absolute values differ moderately, the observed abundance pattern looks very similar for all stars. In general, abundance ratios are more robust than absolute values because they are nearly independent of the EM. We already mentioned that while low activity stars show the FIP

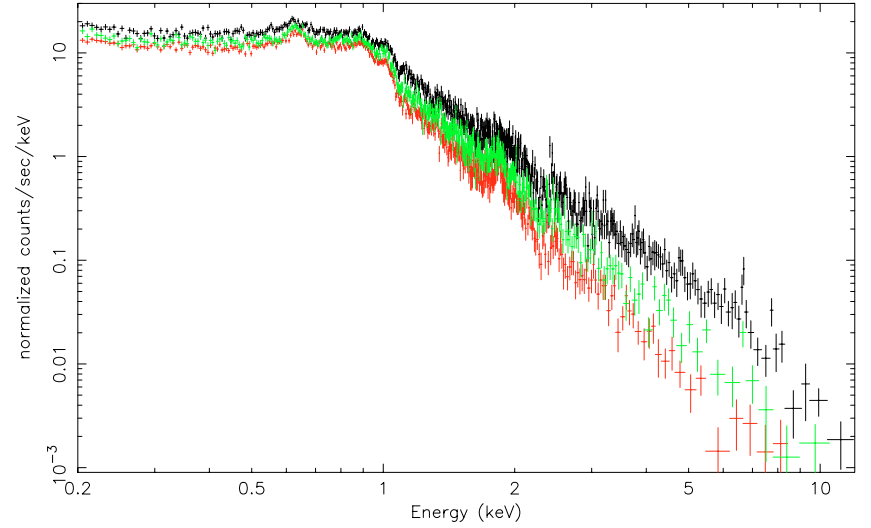

Fig. 6. Spectra of AT Mic during different states of activity, PN data from selected time-intervals as defined in Table 3 (high: black, medium: green, qq: red). (Colour figure in electronic version.)

effect more active stars have shown none or an inverse FIP effect (IFIP) (Brinkman et al. 2001; Audard et al. 2003). As a major trend the inverse FIP effect is clearly present in all analysed sources but individual elements show occasional deviations, e.g. carbon, which is mainly determined by the $\mathrm{C}$ VI line at the low energy end of the RGS. The IFIP effect is detected with all instruments; however, the strength of the IFIP or abundances of individual elements do vary. This can be mainly attributed to instrumental properties, e.g. the often low abundance of sulfur in RGS fits. Here the detectable lines lie at the high energy end of the detector where the effective area is quite small. The inverse FIP effect is strongest for the most luminous source AT Mic and appears to be correlated with activity. Taking the abundance ratio $\mathrm{Ne} / \mathrm{Mg}$ as an indicator of the strength of the IFIP effect (since these elements have the largest difference in FIP), we find the largest ratio for AT Mic, intermediate values for AD Leo and EQ Peg and the lowest ratio is found for EV Lac which also exhibits the lowest absolute abundance values. The same trend is observed for other ratios like $\mathrm{Ne} / \mathrm{Fe}$ or $\mathrm{O} / \mathrm{Fe}$ and likewise in the results obtained with the MOS detector.

\subsection{Flare-related changes}

Significant variations in the abundance pattern were not detected with the EPIC data. We also searched for variations with RGS data, which suggests an enhancement of low FIP elements like iron during strong flares, most prominent in the large flare on AT Mic when compared to the quasi-quiescent phase. While the elements with intermediate FIP show no clear trend, the high FIP element neon may also be somewhat enhanced, a trend already found by Raassen et al. (2003). We caution, however, that the measurement errors are large and that a constant abundance pattern is also acceptable within the $90 \%$ confidence range derived from the model. This effect is still weaker or undetected for the other sample stars.

In contrast to abundances, emission measures and temperatures of the coronal plasma change significantly. As an example we show the PN spectrum of the AT Mic observation in Fig. 6, separated into the three phases of activity. Not only does the 
plasma emission measure increase, but the spectra also harden towards higher activity as more and more emission is found in the higher temperature components during flaring. This is also seen in the growth of the iron lines at $6.7 \mathrm{keV}$, which are predominantly formed at temperatures above $40 \mathrm{MK}$.

A quantitative study of the temporal evolution of plasma temperature during flaring was carried out via the emission measure distribution (EMD). Because the most significant changes are expected to be in the high temperature components of the plasma, we used PN data to study the changes of the EMD. For this purpose we use the 6-T model, and keep the abundances fixed at the values as determined from the total observation to derive the EMDs for the different phases of activity. The first two components represent the cool plasma (2-5 MK), followed by the medium temperature plasma (5-20 MK) and the last two account for the hot plasma (20-70 MK).

The observed sources vary in terms of X-ray luminosity and its increase during flaring. The largest increase is found for the most luminous source AT Mic, where the X-ray luminosity during the flare peak phase reaches $\sim 44 \times 10^{28} \mathrm{erg} \mathrm{s}^{-1}$; the source brightened by nearly a factor of two for about an hour. AD Leo, EQ Peg and EV Lac are about half an order of magnitude X-ray fainter than AT Mic. A graphical representation of the results on the EMDs of the lower activity stars separated into two different phases of activity (three for the most active source AT Mic) is shown in Fig. 7.

The weakest flaring activity is found on EQ Peg, with changes in EM affecting mainly the medium temperature component. The summed EM of the cool component is constant within errors whereas the medium component increases significantly; typical flare temperatures are around 10-15 MK. The hot component is only marginally detected,contributing only $3 \%$ to the EM, and does not appear to be significantly affected by flaring. An intermediate case is observed for AD Leo and EV Lac; here the cool components again stay nearly constant, the medium components increase significantly and the hot component also increases during flaring. The flare temperatures are in the $15-40 \mathrm{MK}$ range. Remarkably the existence of two more separated parts of the EMDs derived from PN data appears also in quasi-quiescence and is similarly found on other temperature grids. However, the respective plasma contributes only a few percent to the total emission measure and the EMDs derived from MOS data are likewise more compact in the sense that the plasma appears to be more concentrated at medium temperatures.

The strongest flaring is observed on AT Mic; here the cooler plasma below the main peak at $\sim 7 \mathrm{MKs}$ is nearly unaffected by the flaring. The main increase is due to the hottest plasma components and large amounts of additional plasma were detected during flaring at temperatures between $15-60 \mathrm{MK}$. In the low state a second weaker peak is visible in the EMD between 25 and $30 \mathrm{MK}$, followed by a steep decline towards higher temperatures, indicating no significant amount of plasma above $40 \mathrm{MK}$. With intermediate conditions during the medium activity phase the plasma distribution during the large flare exceeds $60 \mathrm{MK}$. The contribution of the hot component to the EMD during the flare is around $25 \%$. The influences of possible changes of the abundance pattern on the EMD are minor and we find
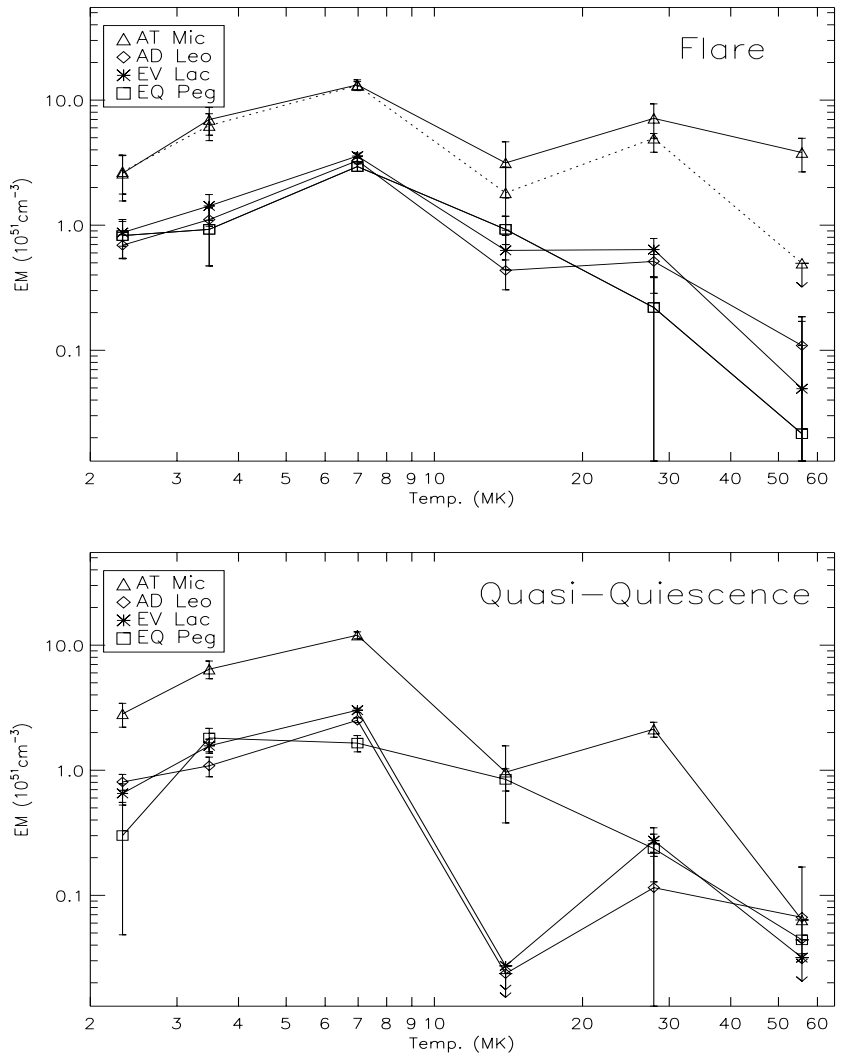

Fig. 7. EMDs of our sample M dwarfs in different states of activity derived from PN data. The upper plot shows the flaring phase, for AT Mic the medium activity phase is additionally plotted as a dashed line while the lower plot shows the quasi-quiescent state. Undetected components are plotted with their upper limits, denoted by downward arrows.

that the basic behaviour and shape of the EMD is comparable for models with free or fixed abundances.

Our analysis of the EMDs shows that flaring does not affect the cool plasma in a significant way. The shape of the EMDs are quite alike at cooler temperatures for all stars and peak at temperatures around 7-8 MK whereas the absolute value correlates with X-ray luminosity. The main influence during flares is found on the amount and temperature of the hotter plasma: stronger flaring and more active stars produce more and hotter plasma. As to the emission of the stars in the quasi-quiescent state a relation between X-ray luminosity and average plasma temperature is suggested while the overall shape of the EMDs is again similar. Only the distribution of binary system EQ Peg, whose EMD is created by two active and slightly different stars (see Robrade et al. 2004), appears broader in all detectors. A hot tail is quite common, sometimes with a second smaller peak, extending to $\sim 30 \mathrm{MK}$ in the EMD. This may indicate the existence of separated plasma components at different temperatures which are located in different coronal structures even in quasi-quiescence, i.e. the main part of the plasma in cooler, more quiescent structures and a smaller amount in hotter and more active structures. These structures would also be a natural explanation for the constant flickering observed in the light curves. 


\subsection{Loops}

Assuming quasi-static loop-like structures as building blocks for the coronae of $\mathrm{M}$ dwarfs the derived EM distributions suggest coronae composed out of different loop components with different temperatures. Using the RTV scaling law (Rosner et al. 1978) for simple loops in hydrostatic equilibrium and with constant pressure and cross-section, and the densities derived by Ness et al. (2004), we are able to calculate the size of the loops. In cgs units we obtain:

$n_{\mathrm{e}} L=1.3 \times 10^{6} T_{\max }^{2}$,

with the electron density $n_{\mathrm{e}}$, the loop top temperature $T_{\max }$ and the (semi) loop length $L$. The typical electron densities of our sample stars in $\log \left(n_{\mathrm{e}}\right)$ as measured from the O VII triplet are around 10.5, and for the Ne IX triplet in the range 11.1-11.5, but for Ne IX the errors are about 0.5 translating into a factor of three in linear units. The O VII triplet traces plasma around $2 \mathrm{MK}$, the Ne IX triplet plasma around $4 \mathrm{MK}$, thus indicating denser plasma at higher temperatures. However both triplets trace only the relatively cool plasma since we find average coronal temperatures of about $10 \mathrm{MK}$ for the less active stars and $15 \mathrm{MK}$ for AT Mic from the EMDs, in good agreement with the values calculated with a scaling law correlating X-ray luminosity and stellar radius $\left(R_{*}\right)$ with average temperature (Ness et al. 2004). While the assumptions of quasi-static loops and the measured densities can be well justified for the cooler and the quiescent components, results for the hotter plasma should be taken as upper limits, especially when taking into account the plausible higher density of the hotter plasma.

Assuming stellar radii of $0.35 / 0.3$ solar radius for spectral type M3.5/M4.5 and for the binaries for simplicity one dominant component, we determine loop lengths for the coolest plasma $(2-5 \mathrm{MK})$ below $0.01 R_{*}$ on all stars. Taking the mean plasma density derived from neon, the loop lengths are about $0.03-0.07 R_{*}$ for the average coronal temperature of our stars and increase up to $0.3 R_{*}$ for the hotter plasma components. Ness et al. (2004) find the density of the hotter plasma consistent with the low density $\operatorname{limit}\left(\log n_{\mathrm{e}} \approx 12.7\right)$ for the used line ratios of Fe XXI, so an higher density as the one derived from Ne IX in the hotter loops would further scale down the determined loop length according to the above scaling law; instead of an increased loop size, an increased density also can explain the observed temperatures. Repeating the calculations with the low density limit of Fe XXI, we derive lower limits for the length of the hotter loops, which are scaled down by a factor of 20 compared to the above derived results. Therefore we conclude that the dominating coronal structures for the cool plasma are very small, i.e. $L<0.01 R_{*}$, for the medium temperature plasma small in the sense that $L<0.1 R_{*}$ and still compact ( $L \lesssim 0.3 R_{*}$ ) for the hotter plasma observed here. Favata et al. (2000a) studied AD Leo data from various missions, analysed decay times of flare loops and likewise found its corona to be compact, even for flare structures.

In this picture the cool emission is produced in relatively small loops nearly unaffected by flaring, while medium and hot temperature loops are larger, probably have higher densities and are more numerous on more active stars or during flares.

\subsection{Comparison with other results}

We compare our results with other measurements and earlier works to check the consistency of the derived results and to investigate long-term behaviour. We find that the results presented here agree well with other analyses, which often used different techniques or dealt with individual objects only, leading to a rather consistent picture of the X-ray properties of active $\mathrm{M}$ dwarfs as a class and demonstrate the robustness of the results.

Comparison of the measured fluxes of our sample stars with e.g. the ROSAT all-sky survey values (Hünsch et al. 1999) shows agreement to within a factor less than two. This is of the same order as the variations found during the moderate activity in the XMM-Newton observations, demonstrating the stability of the X-ray emission. Favata et al. (2000a) studied the quasi-quiescent corona of $\mathrm{AD}$ Leo from various missions and found the X-ray luminosity to be remarkable constant with variations less than a factor of two, suggesting the absence of strong cyclic variations; our results agree well, expanding the time period of the observations to over 20 years. The X-ray luminosity of EQ Peg determined in this work agrees likewise with the measurements made with Einstein more than 20 years ago. Combined, these findings establish long term stability of the quasi-quiescent corona for active $\mathrm{M}$ dwarfs in general.

Comparing our results with previous analysis of the XMM-Newton data, our AD Leo results are in good agreement with those obtained by Besselaar et al. (2003), who also applied the global fitting approach, especially when considering the different spectral code and model, atomic database and of course the X-ray dataset used in the analyis. The same is true for the AT Mic results presented in Raassen et al. (2003) with a similar analysis; note that errors stated in these publications are only $1 \sigma$. Discrepancies concerning the absolute values of emission measure and abundances are explained by their mutual interdependence, and a combination, e.g. the product, of these properties is in much better agreement. Concerning the shape of the DEM, which is determined there with polynomial methods from MOS and RGS data, results are comparable with our EMD based on multi-temperature fits of PN data.

Maggio et al. (2004) presented a line-based analysis of AD Leo data taken with Chandra LETGS and also found the inverse FIP effect. However, they determined the iron abundance from the line-to-continuum ratio in the low-count spectrum to be almost as high as that in the solar photosphere. Consequently, nearly all other elements, which are scaled with the iron abundance, appear to be supersolar. On the other hand, Favata et al. (2000a) determined the iron abundance from ASCA data to $\sim 0.2$ solar, low but comparable to our EPIC values. While the abundances determined with medium resolution spectroscopy are apparently often low, the results obtained with the global fits of high resolution data in our work or in Besselaar et al. (2003) appear more reliable. We also point out that our joint fit approach, which uses a data selection comparable to Audard et al. (2004), who obtained consistent results on the abundances with line-based and global models applied to high resolution X-ray spectra, results in slightly increased but still significant subsolar abundance values. 
Whereas the global metallicities were not determined uniquely in the various analysis, the inverse FIP effect is consistently found in all analysis of active $\mathrm{M}$ dwarfs, despite the fact that different spectral models and atomic codes were applied to medium and high resolution X-ray data, demonstrating that the inverse FIP effect is certainly a robust result.

\section{Summary and conclusions}

We have carried out a comparative analysis of XMM-Newton observations of $\mathrm{M}$ dwarfs with spectral type M3.5-M4.5, i. e., AT Mic, EQ Peg, AD Leo and EV Lac. Light curves revealed frequent flaring of all observed sources on different timescales and with various strengths of the individual flares. While AD Leo, EQ Peg and EV Lac are comparable with respect to activity and X-ray brightness, the probably younger system AT Mic is half an order of magnitude X-ray brighter. From X-ray spectroscopy, abundance patterns and emission measure distributions were derived for the sample stars and for different states of activity. We find that many X-ray quantities, like the coronal temperature structure and the abundance pattern of the analysed $\mathrm{M}$ dwarfs are very similar despite the fact that the X-ray luminosity and flaring behaviour differs significantly.

All analysed $\mathrm{M}$ dwarf coronae show the inverse FIP effect, demonstrated in this work for the first time for EQ Peg and EV Lac, establishing this effect for the coronae of active $\mathrm{M}$ dwarfs as a class. The determined abundances are lower than solar photospheric abundances; however, the few available measurements of stellar photospheric metallicities for $\mathrm{M}$ dwarfs also point to subsolar values, comparable with our coronal measurements. This suggests that a strong coronal metal abundance deficiency may actually not be present in our sample stars. Properties like X-ray brightness, temperature of the flare plasma and thus hardness of the X-ray spectrum are highest for the most active source AT Mic, and increase during flares for all observed sources, suggesting a correlation between these properties and activity. The strength of the inverse FIP effect is also greatest in AT Mic, but does not increase during flaring. Time-resolved abundance analysis suggests that preferably low FIP elements are enhanced during flares, but this finding is not statistically significant.

We find from the derived EMDs of the stars that the plasma temperature structures of the coronae are similar, with a maximum at $7-8 \mathrm{MK}$ and a hot tail with a stronger contribution of hotter plasma in the X-ray brighter stars. The emission measures of the hotter plasma components increase significantly during flares and higher temperatures are reached in stronger flares, while the cool plasma is only marginally affected by flaring. Assuming that the coronae are composed of loop-like structures, this indicates a distribution of structures in which the cooler plasma is confined in many smaller loops while other, presumably larger and denser loops, dominate the additional hot emission generated by flare events. The coronae are found to be compact, i.e. the largest loop lengths are smaller than one third stellar radius.

From comparison with older measurements we conclude that the global X-ray properties show long-term stability while short time-scale behaviour is characterised by frequent variations on top of a level of "baseline activity". With the XMM-Newton data we are able to investigate the X-ray properties of stellar coronae to a previously unknown precision. As these observations provide only a snapshot in the life of a few M dwarfs, larger samples or longer observations may show more extreme or different phenomena. Nevertheless, the consistency and congruence makes our results appear typical for active $\mathrm{M}$ dwarfs.

Acknowledgements. This work is based on observations obtained with XMM-Newton, an ESA science mission with instruments and contributions directly funded by ESA Member States and the USA (NASA). This research has made use of the SIMBAD database, operated at CDS, Strasbourg, France (http://simbad.u-strasbg . fr).

We thank the referee for useful comments.

J.R. acknowledges support from DLR under 50OR0105.

\section{References}

Allende Prieto, C. A., Lambert, D. L., \& Asplund, M. 2001, ApJ, 556, L63

Anders, E., \& Grevesse, N. 1989, Geochim. Cosmochim. Acta, 53, 197

Arnaud, K. A. 1996, ASP Conf. Ser., 101, 17

Audard, M., Güdel, M., Sres, A., et al. 2003, A\&A, 398, 1137

Audard, M., Telleschi, A., Güdel, M., et al. 2004, ApJ, 617, 531

Barrado y Navascues, D., Stauffer, J. R., Song, I., \& Caillault, J.-P. 1999, ApJ, 520, L123

van den Besselaar, E. J. M., Raassen, A. J. J., Mewe, R., et al. 2003, A\&A, 411, 587

Brinkman, A. C., Behar, E., Güdel, M., et al. 2001, A\&A, 365, L324

Delfosse, X., Forveille, T., Perrier, C., \& Mayor, M. 1998, A\&A, 331, 581

Ehle, M., Breitfellner, M., Gonzales Riestra, M., et al. 2003, XMMNewton User's Handbook

Ehle, M., Pollock, A. M. T., Talavera, A., et al. 2004, User's Guide to XMM-Newton Science Analysis System

Favata, F., Reale, F., Micela, et al. 2000a, A\&A, 353, 987

Favata, F., Micela, G., \& Reale, F. 2000b, A\&A, 354, 1021

Feldman, U., Seely, J. F., Doschek, G. A., et al. 1995, ApJ, 446, 869

Fuhrmeister, B., Schmitt, J. H. M. M., \& Wichmann, R. 2004, A\&A, 417, 701

Grevesse, N., \& Sauval, A. J. 1998, Space Sci. Rev., 85, 161

Güdel, M., Audard, M., Briggs, K., et al. 2001, A\&A, 365, L336

Güdel, M., Audard, M., Kashyap, V. L., et al. 2003, ApJ, 582, 423

Güdel, M., Audard, M., Reale, F., et al. 2004, A\&A, 416, 713

Holweger, H. 2001, AIPC, 598, 23

Hudson, H. S. 1991, SoPh, 133, 357

Hünsch, M., Schmitt, J. H. M. M., Sterzik, M. F., et al. 1999, A\&AS, 135,319

Kahn, S. M., Linsky, J. L., Mason, K. O., et al. 1979, ApJ, 234, L111

Kashyap, V. L., Drake, J. J., Güdel, M., \& Audard, M. 2002, ApJ, 580, 1118

Katsova, M. M., Livshits, M. A., \& Schmitt, J. H. M. M. 2002, ASP Conf. Proc., 177, 515

Kenyon, S. J., \& Hartmann, L. 1995, ApJS, 101, 117

Kirsch, M. G. F., Altieri, B., Chen, B., et al. 2004

[arXiv:astro-ph/0407257]

Laming, J. M., Drake, J. J., \& Widing, K. G. 1995, ApJ, 443, 416

Maggio, A., Drake, J. J., Kashyap, V., et al. 2004, ApJ, 613, 548 
Naftilan, S. A., Sandmann, W. S., \& Pettersen, B. R. 1992, PASP, 104, 1045

Ness, J.-U., Schmitt, J. H. M. M., Burwitz, V., et al. 2002, A\&A, 394, 911

Ness, J.-U., Güdel, M., Schmitt, J. H. M. M., et al. 2004, A\&A, 427, 667

Parker, E. N. 1988, ApJ, 330, 474

Proquet, D., Mewe, R., Dubau, J., et al. 2001, A\&A, 376, 1113

Raassen, A. J. J., Mewe, R., Audard, M., \& Güdel, M. 2003, A\&A, 411, 509

Robrade, J., Ness, J.-U., \& Schmitt, J. H. M. M. 2004, A\&A, 413, 317

Rosner, R., Tucker, W. H., \& Vaiana, G. S. 1978, ApJ, 220, 643

Sanz-Forcada, J., Maggio, A., \& Micela, G. 2003, A\&A, 408, 1087
Schmitt, J. H. M. M., \& Rosso, C. 1988, A\&A, 191, 99

Schmitt, J. H. M. M., \& Liefke, C. 2004, A\&A, 417, 651

Schmitt, J. H. M. M., Fleming, T. A., \& Giampapa, M. S. 1995, ApJ, 450, 392

Smith, R. K., Brickhouse, N. S., Liedahl, D. A., \& Raymond, J. C. 2001, ASP Conf. Ser., 247, 161

Sciortino, S., Maggio, M., Favata, F., \& Orlando, S. 1999, A\&A, 342, 502

Stauffer, J. R., \& Hartmann, L. W. 1986, ApJS, 61, 531

Vaiana, G. S., Cassinelli, J. P., Fabbiano, R., et al. 1981, ApJ, 244, 163

Zboril, M., Byrne, P. B., \& Rolleston, W. R. J. 1998, MNRAS, 299, 753

Zuckerman, B., Song, I., Bessell, M. S., \& Webb, R. A., ApJ, 562, L87 